\title{
The edges of the stellar populations of early type spirals as probed by their radial brightness profiles
}

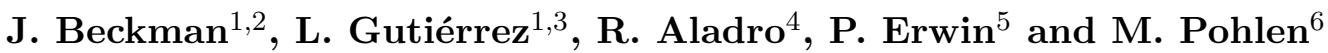 \\ ${ }^{1}$ Instituto de Astrofísica de Canarias, Tenerife, Spain. jeb@iac.es \\ ${ }^{2}$ Consejo Superior de Investigaciones Científicas, Spain \\ ${ }^{3}$ Universidad Nacional Autónoma de México, Ensenada, México. leonel@astrosen.unam.mx \\ ${ }^{4}$ Universidad de La Laguna, Tenerife, Spain. rebeca_aladro@yahoo.es \\ ${ }^{5}$ Max Planck Institut für Astrophysik, Garching, Germany. erwin@mpe.mpg.es \\ ${ }^{6}$ Kapteyn Instituut, Rijksuniversiteit Groningen, Netherlands. pohlen@astro.rug.nl
}

\begin{abstract}
We have derived radial sky-subtracted surface brightness profiles of a sample of 42 face-on nearby early-type spiral galaxies, classified as unbarred, from SDSS data as well as our own observations (INT/WFC) at La Palma. A key objective was to derive the fraction of truncated profiles. We find that only a small minority $7 \%$ of the galaxies are obviously truncated within the limits of the measured surface brightness range, while some $20 \%$ show exponential disc profiles but with a shallower inner portion near the centre: classical "Freeman Type II" profiles (Freeman, 1970). Half of the discs show a single unbroken exponential ("type I") brightness profile with no evidence of truncation out to the edge of detectability, while the remainder show "antitruncations": external profiles less steep than those of the internal disc (Type III). A general profile classification scheme based on these and other recent related measurements is presented, and will be of use in probing disc formation scenarios.
\end{abstract}

Keywords. Galaxy disks, brightness profiles, truncations, antitruncations.

\section{The sample}

The sample presented here is of 42 early type galaxies chosen as their classification is that of "unbarred" (although a few of them may in fact be very weakly barred). The other criteria were that they should be nearby and well resolved, and face-on, with inclination angle less than 65 degrees. For 15 of these we used SDSS images in the r' band to make the profiles, and the remaining 27 were observed by ourselves in the $r$ band with the Wide Field Camera on the 2.5m Isaac Newton Telescope (La Palma). Very careful star masking and sky subtraction were followed by the extraction of radial surface brightness profiles which we present here down to a $3 \sigma$ limiting brightness level.

\section{Frequency of profile types and conclusions}

In this presentation we will not attempt to derive a frequency distribution of the profile types. In Pohlen \& Trujillo (2006) an equivalent distribution was given for some 90 late type discs, and a compilation for early type barred galaxies with those from Pohlen and Trujillo was presented in Beckman et al. (2006). However as we have not finished our analysis of the present sample it would not be correct to include the assignments shown in this paper in an overall compilation, including unbarred early type discs, the subject of our presentation here. Only one comment is relevant, the number of type II profiles (which would include classical truncations) is clearly much lower for early type galaxies than for late type and the number of type III profiles is higher. 


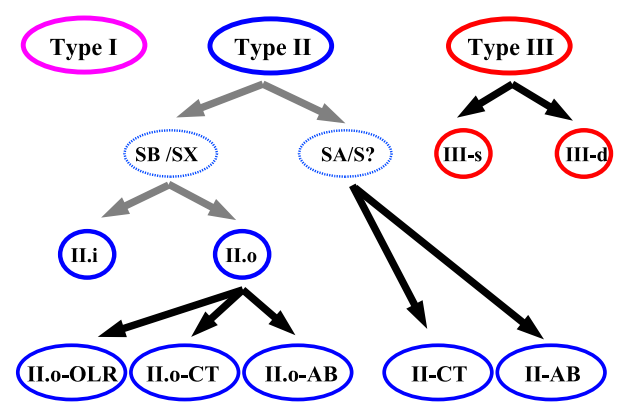

Figure 1. This scheme shows the three basic radial surface brightness profile classes (Type I, single exponential, Type II, broken exponential with outer slope steeper, Type III, broken exponential with outer slope shallower). For a detailed description of the sub-classes in Type II, see Pohlen \& Trujillo (2006) from which the diagram is taken.
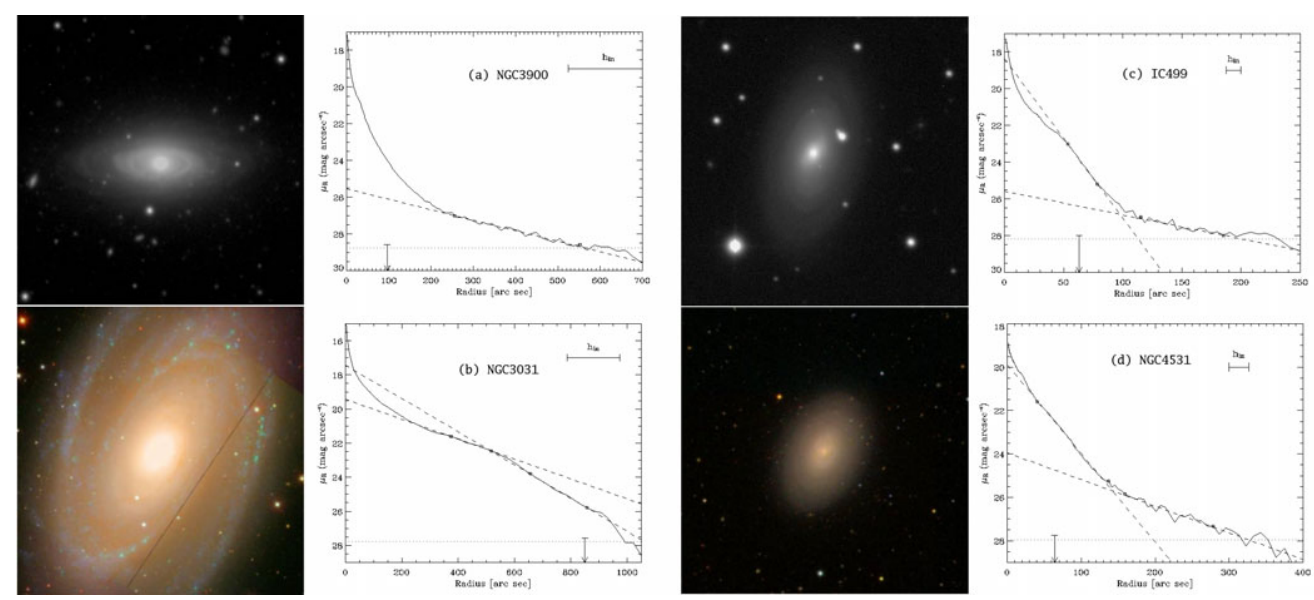

Figure 2. Some examples of differente profiles: (a) Type I, (b) Type II-CT, (c) Type II-CT + III, (d) Type III.

Before arriving at a firm hypothesis on how galaxy discs form and evolve, careful and systematic observational study of their surface brightness profiles is still necessary, as our perspectives on these observations have changed rapidly in very recent years, and are still changing as new results come in.

\section{References}

Beckman, J.E., Erwin, P., Pohlen, M., Gutiérrez, L., Aladro, R., \& Trujillo, I. "The external zones of spiral galaxies: Truncations, No Truncations and Antitruncations". In "Mapping the Galaxy and Nearby Galaxies," Ishigaki Island, Japan, June 2006. Ed. K.Wada . (In press).

Erwin, P., Beckman, J.E., \& Pohlen, M. 2005, ApJ, 626L, 81

Erwin, P., Pohlen, M., \& Beckman J.E. 2007a, A $\mathscr{G A}$ (In preparation)

Erwin, P., Gutiérrez, L., Pohlen, M., Beckman, J.E., \& Aladro, R. 2007b, A\&A (in preparation).

Freeman, K.C. 1970, ApJ, 160, 811

Pohlen, M., Dettmar, R.-J., Lütticke, R., \& Aronica, G. 2002, A\&A 392, 807

Pohlen, M. \& Trujillo, I. 2006, A\&A 454,759

van der Kruit, P.C. \& Searle, L. 1981, A\&A 95, 116 